\title{
COMPORTAMIENTO OBSTETRICO DE MUJERES MENORES DE 20 AÑOS COMPARADO CON EL DE MUJERES DE OTRAS EDADES
}

Trabajo parcialmente patrocinado por la Asociación Colombiana de Facultades de Medicina, División de Estudios de Población. - Presentado en el IX Congreso Colombiano de Obstetricia y Ginecología. - Bucaramanga, Diciembre, 1971.

\author{
Dr. Jaime Botero Uribe* \\ Dr. Julio César Tuberquia C.** \\ Sr. Marcelo Huerta***
}

Con la ayuda del Sr. Francisco Cifuentes (Ascofame).

\section{Introducción}

En Colombia hay 4'375.000 mujeres de 15 a 54 años, que constituyen el $24,2 \%$ de la población total del país. Se considera que la etapa reproductiva de la mujer en Colombia está comprendida entre esas edades. El $56,5 \%$ de estas mujeres viven en la zona urbana. El grupo de mujeres entre 15 y 19 años es el más numeroso y representa el $22,1 \%$ del total, es decir, la quinta parte o sea aproximadamente un millón (1).

Claman y colaboradores (2) en un trabajo hecho en Columbia Británica, Canadá, en mujeres embarazadas menores de 16 años, encontraron que el embarazo se ha triplicado en ese grupo de edad en la década de 19501969 y que la edad de la menarca ha disminuído de 17 a 13 en los últimos 100 años.

Russel (3) encontró que el embarazo en niñas de 15 ha aumentado en Inglaterra entre 1956 y 1966 de 169 a 1.288 por año, es decir, de un
0,8 a un 3,28 por mil. Sin embargo la tasa de nacimientos ha bajado desde 1964.

El embarazo en las edades extremas de la vida reproductiva de la mujer significa un riesgo. Esto ha sido estudiado en otros países pero no en Colombia; al menos no se conoce ninguna publicación a este respecto. La literatura revisada demuestra que en mujeres embarazadas de menos de 20 años es más frecuente la toxemia $(2,4,7,8,9,10,12,15)$, la prematuridad $(2,5,9,10,12,13$, $15)$, los niños de bajo peso $(7,8$, $13)$, el exceso de peso en la paciente $(2,12)$, el trabajo prolongado (4, $7,8,14)$, la pelvis estrecha (4), la mortalidad neonatal e infantil (5),

* Profesor, Departamento de Obstetricia y Ginecología, Facultad de Medicina, Universidad de Antioquia. Ap. Aéreo 1226 - Medellín, Colombia.

** Profesor Auxiliar, Departamento de Obstetricia y Ginecología, Facultad de Medicina, Universidad de Antioquia.

*** Estadístico. Profesor de la Escuela Nacional de Salud Pública. Universidad de Antioquia. 
la consulta prenatal deficiente (2) el hogar disuelto (13), el suicidio (11), los hijos naturales a repetición (6). Las nulíparas de más de treinta años tienen una mortalidad perinatal más alta y una mayor incidencia de anomalías congénitas (14).
A pesar de lo anterior, cuando las mujeres adolescentes son cuidadosamente controladas, como lo hicieron Sarrel y Klerman (16), la incidencia de complicaciones baja en forma dramática; así se observa en el siguiente cuadro comparativo:

\begin{tabular}{lccc}
\hline Autor & Toxemia & Prematuridad & Mortalidad \\
\hline & $\%$ & $\%$ & $\%$ \\
Marrchetti y Menaker & 19,7 & 14,8 & 3,8 \\
Aznar y Benett & 9,8 & 18,7 & 5,0 \\
Morrison & 21,0 & 16,0 & 3,8 \\
Poliakoff & 17,7 & 17,4 & 5,9 \\
Sarrel y Klerman & 5,0 & 10,8 & 0,8 \\
\hline
\end{tabular}

\section{Objetivos}

El presente trabajo se propuso:

1. Determinar algunas variables obstétricas en las mujeres que asistieron al servicio de maternidad del Hospital Universitario San Vicente de Paúl de Medellín, entre Abril 1ํ de 1969 y Abril 30 de 1971.

2. Buscar algunas diferencias en el comportamiento obstétrico entre mujeres menores de 20 años y las de otros grupos de edades.

3. Analizar algunas variables de la patología obstétrica en esos mismos grupos de pacientes.

4. Determinar la mortalidad fetal en relación con la edad de la madre y el tipo de parto.

\section{Material y Métodos}

Se analizaron 7.897 historias de pacientes admitidas al servicio obstétrico en el período mencionado, después de trasladarlas a tarjetas IBM. Se revisó una pequeña muestra de tarjetas e historias para ver su correspondencia. Se hizo un plan de tabulación con un total de 15 cuadros, utilizando 10 variables para cruces entre ellas, hasta un máximo de tres variables por cuadro. La variable independiente básica para esta investigación fue la edad de la embarazada, con la cual se establecieron tres grispos de mujeres:

Grupo A, menores de 20 años.

Grupo B, de 20 a 34 años.

Grupo C, de 35 y más años.

El grupo A estaba constituído casi en su totalidad por mujeres entre 15 y 19 años; sólo se encontraron en un año 6 mujeres de 14

Las demás variables fueron: 1. paridad; 2. abortos; 3. toxemia; 4. estado de las membranas; 5 . duración del primer período del parto; 6. duración del segundo período del parto; 7. tipo de parto; 8. peso del niño al nacer; 9. mortalidad fetal; 10. infección puerperal.

Para la definición de muerte fetal adoptamos la que recomienda la $\mathrm{Or}$ ganización Fanamericana de la Salud (17): "muerte fetal precoz, aquella que ocurre antes de las 20 semanas completas de gestación; intermedia, la que ocurre después de las 20 pero 
antes de las 28 semanas, y tardía de 28 semanas completas de gestación y más".

Para las tabulaciones se usó el programa Mini-Tab frecuencias y cuadros que tiene la Asociación cle Facultades de Medicina en la Unidad de Valoración, usando el computador IBM 3-60-40.

\section{Análisis de los Resultados}

1. Distribución de las pacientes por grupos de edad. La mayor parte de las pacientes estudiadas, el $65 \%$, estaba en el grupo de 20 a 34 años. Un $19 \%$ eran menores de 20 y cerca del $17 \%$ eran mayores de 35 . (Cuadro No 1).

Cuadro No ।

DISTRIBUCION DE LAS PACIENTES POR GRUPOS DE EDAD

H.U.S.V.P. ABRIL 1.969 - ABRIL I.97I. MEDELLIN.

\begin{tabular}{|c|c|c|c|c|c|c|}
\hline DISTRIBUCION & POR & GRUPOS & $\mathrm{DE}$ & EDAD & Noㅇ & $\%$ \\
\hline GRUPO & $A$ & menores & de & 20 años & 1.465 & 18.6 \\
\hline GRUPO & B & de 20 & $a$ & 34 años & 5.082 & 64.7 \\
\hline GRUPO & C & de 35 & $y$ & mas años & 1.315 & 16.7 \\
\hline TOTAL & $D E$ & $P A C \mid E N^{-}$ & $E S$ & & 7.862 & 100.0 \\
\hline
\end{tabular}

2. Paridad de las pacientes. En el Cuadro NN 2 se aprecia la paridad del grupo en el cual se analizó esta variable, que comprendía 4.831 pacientes. Se observa que la mitad de ellas había tenido entre 0 y 1 parto y que un $60 \%$ había tenido hasta dos.

3. Relación entre partos y abortos. Sobre un total de 7.842 casos analizados para esta variable, el $28 \%$ corresponden a abortos, el $71 \%$ a partos y el $1 \%$ a casos desconocidos. La definición de aborto incluyó la muerte fetal precoz e intermedia.
4. Incidencia de toxemia. La incidencia de toxemia fue de $6,4 \%$ para el período estudiado, (Cuadro № 3) lo cual significa un discreto aumento sobre lo encontrado en el estudio de mortalidad perinatal realizado en 1959 en el mismo hospital, que fue de $6,1 \%$ (18). La toxemia moderada ocurrió en un $71 \%$ de los casos; en el $24 \%$ fue severa y en el $4,6 \%$ hubo eclampsia. (Cuadro No 4).

Al analizar el Cuadro № 5, que muestra las tasas de incidencia de toxemia según los grupos de edad, se 
Cuadro $\quad N \circ 2$

DISTRIBUCION DE LAS PACIENTES POR PARIDAD

H.U.S.V.P. ABRIL I.969 - ABRIL I.97I MEDELLIN

\begin{tabular}{c|c|c}
\hline PARIDAD & No & $\%$ \\
\hline 0 & 1.420 & 29 \\
1 & 921 & 19 \\
2 & 574 & 11 \\
3 & 416 & 9 \\
4 & 313 & 7 \\
5 & 273 & 7 \\
6 & 200 & 4 \\
7 & 162 & 3 \\
8 y más & 552 & 10 \\
\hline
\end{tabular}

CUADRO № 3

INCIDENCIA DE TOXEMIA

H.U.S.V.P. - ABRIL 1969 - ABRIL 1971 - MEDELLIN

TOTAL DE MADRES ATENDIDAS: 7.862

PRESENTARON ALGUN TIPO DE TOXEMIA: 507 - 6,4\%

encuentra que el grupo B tuvo la ta- comparar el grupo de mujeres de 20 sa más baja: 57 por mil madres, a 34 años con el de mujeres más jómientras que el de madres menores venes, se observa que la relación es de 20 años mostró la más alta: 87 por mil. En el grupo de madres mayores de 35, la tasa fue de 69. Al de $1: 1,5$, y al compararlo con el cle las mayores de 35 años, la relación es de 1:1,2. Ambas diferencias son estadísticamente significativas. 
TIPOS DE TOXEMIA SEGUN EDAD DE LAS MADRES

- EXPRESADOS EN \%

H.U.S.V.P. ABRIL 1.969 - ABRIL 1.971 MEDELLIN

\begin{tabular}{|c|c|c|c|c|}
\hline GRUPOS & MODERADA & SEVERA & ECLAMPSIA & $\begin{array}{l}\text { HIPERTENSION } \\
\text { Y TOXEMIA }\end{array}$ \\
\hline A & 16.0 & 6.7 & 2.4 & \\
\hline$B$ & 41.0 & 13.6 & 1.8 & 0.6 \\
\hline C & 13.6 & 3.7 & 0.4 & 0.2 \\
\hline \multirow[t]{2}{*}{ TOTAL } & 70.6 & 24.0 & 4.6 & 0.8 \\
\hline & & $1=7.862$ & $\begin{array}{l}A=< \\
B= \\
C=\end{array}$ & $\begin{array}{l}20 \text { años } \\
0-34 \text { años } \\
5 \text { y más años }\end{array}$ \\
\hline
\end{tabular}

Cuadro N2 5

TASAS DE INCIDENCIA DE TOXEMIA SEGUN GRUPOS DE EDAD

H.U.S.V.P. ABRIL 1.969 - ABRIL 1.971 MEDELLIN

\begin{tabular}{c|c|c|c|c|c}
\hline GRUPOS & MODERADA & SEVERA & ECLAMPSIA & $\begin{array}{c}\text { HIPERTENSION } \\
\text { Y TOXEMIA }\end{array}$ & $\begin{array}{c}\text { TASAS POR } \\
\text { MIL MADRES }\end{array}$ \\
\hline A & 55 & 23 & 8 & & 86.7 \\
B & 41 & 14 & 2 & 0.6 & 56.9 \\
C & 52 & 14 & 2 & 0.8 & 69.2 \\
\hline
\end{tabular}

$$
\begin{array}{ll}
N=7.862 & A=<20 \text { años } \\
Z_{\text {Busc }}=>1.96 & B=20-34 \text { años } \\
Z_{\text {Busc }}=>1.96 & C=35 \text { y más años } \\
\mathcal{L}=0.05 &
\end{array}
$$


En el grado de toxemia moderada, se observa que el grupo B tuvo la tasa más baja en relación con los otros dos y que la diferencia es tan marcada, que dá significación estadística al nivel de 0.05. En los otros grados de toxemia, los grupos B y C fueron sensiblemente iguales, observándose en ambos tasas menores que las del grupo A.

5. Estado de las membranas. Esta definición incluyó todo tipo de membranas rotas, tanto artificial como espontáneamente, hasta el fin del primer período del parto. De 4.995 pacientes, se presentaron membranas rotas en 745 , un $15 \%$ del total de casos, lo cual nos parece que representa una incidencia alta. En el Gráfico № 1 se abserva que el grupo de mujeres mayores de 35 años presentó membranas rotas en una mayor pro- porción de casos que los otros dos. Las cifras allí anotadas representan tasas porcentuales. Buscando la significación estadística de este hallazgo por medio del test de las proporciones, con alfa: 0,05, se encontró que al comparar el grupo A con el C la diferencia es significativa. ( $Z$ mayor de 1.96). Lo mismo sucede al comparar el grupo B con el C ( $Z$ mayor de 1.96). En cambio, la diferencia entre los grupos A y B no tiene significación estadística ( $Z$ menor de 1.96).

Indagamos también la asociación existente entre ruptura de membranas y tipo de parto, en los distintos grupos de edades de madres (Cuadro № 6, Gráfica № 2). En el parto espontáneo la ocurrencia de ruptura de membranas fue más frecuente, con fluctuaciones que van del $16 \%$ en el

Grafico № I

\section{PORCENTAJE DE MEMBRANAS ROTAS EN 4.955 CASOS ESTUDIADOS, EN TRES GRUPOS DE EDAD DE LAS MADRES \\ H.U.S.V.P. ABRIL 1.969 - ABRIL I.97I MEDELLIN}

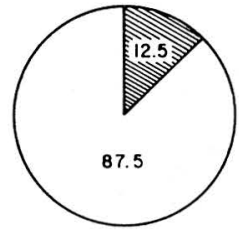

GRUPO A
$A=<20 \Delta \bar{N} O S$

$B=20-34 \Delta A N O S$

$C=35$ Y MAS AÑOS

GRUPO B.

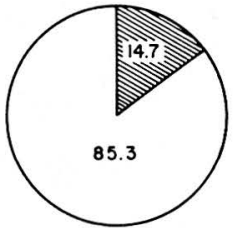

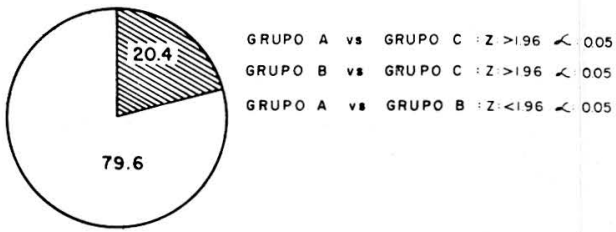

GRUPO C.

No rotas

Rotas 
Cuadro $N \cong 6$

ESTADO DE LAS MEMBRANAS SEGUN EDAD DE LA MADRE Y TIPO DE PARTO - EXPRESADO EN \%

H.U.S.V.P. ABRIL 1.969 - ABRIL 1.971 MEDELLIN

\begin{tabular}{c|c|c|c|c|c|c|c|c|c|c|c}
\hline \multirow{2}{*}{ GRUPOS } & \multicolumn{2}{|c|}{ ESPONTANEO } & \multicolumn{2}{c|}{ FORCEPS } & \multicolumn{2}{c|}{ EXTRACTOR } & \multicolumn{2}{c|}{ CESAREA } & \multicolumn{2}{c|}{ TOTAL } & \multicolumn{2}{c}{ TOTAL } \\
\cline { 2 - 9 } & ROTAS & $\begin{array}{c}\text { NO } \\
\text { ROTAS }\end{array}$ & ROTAS & $\begin{array}{c}\text { NO } \\
\text { ROTAS }\end{array}$ & ROTAS & $\begin{array}{c}\text { NO } \\
\text { ROTAS }\end{array}$ & ROTAS & $\begin{array}{c}\text { NO } \\
\text { ROTAS }\end{array}$ & ROTAS & $\begin{array}{c}\text { NO } \\
\text { ROTAS }\end{array}$ & GENERAL \\
\hline A & 10.1 & 65.8 & 1.9 & 17.1 & & 1.3 & 0.5 & 3.3 & 12.5 & 87.5 & 100 \\
\hline B & 11.8 & 73.3 & 1.2 & 7.3 & 0.5 & 1.9 & 1.1 & 2.9 & 14.7 & 85.3 & 100 \\
\hline C & 16 & 66.6 & 1.2 & 1.9 & 0.7 & 3.6 & 2.5 & 7.5 & 20.4 & 79.6 & 100 \\
\hline
\end{tabular}

Grafico №2

PRESENCIA DE MEMB. ROTAS, SEGUN EDAD DE LA MADRE Y TIPO DE PARTO. CASOS ANAL. 4.955 H.U.S.V.P. ABRIL 1.969 - ABRIL 1.971 MEDELLIN

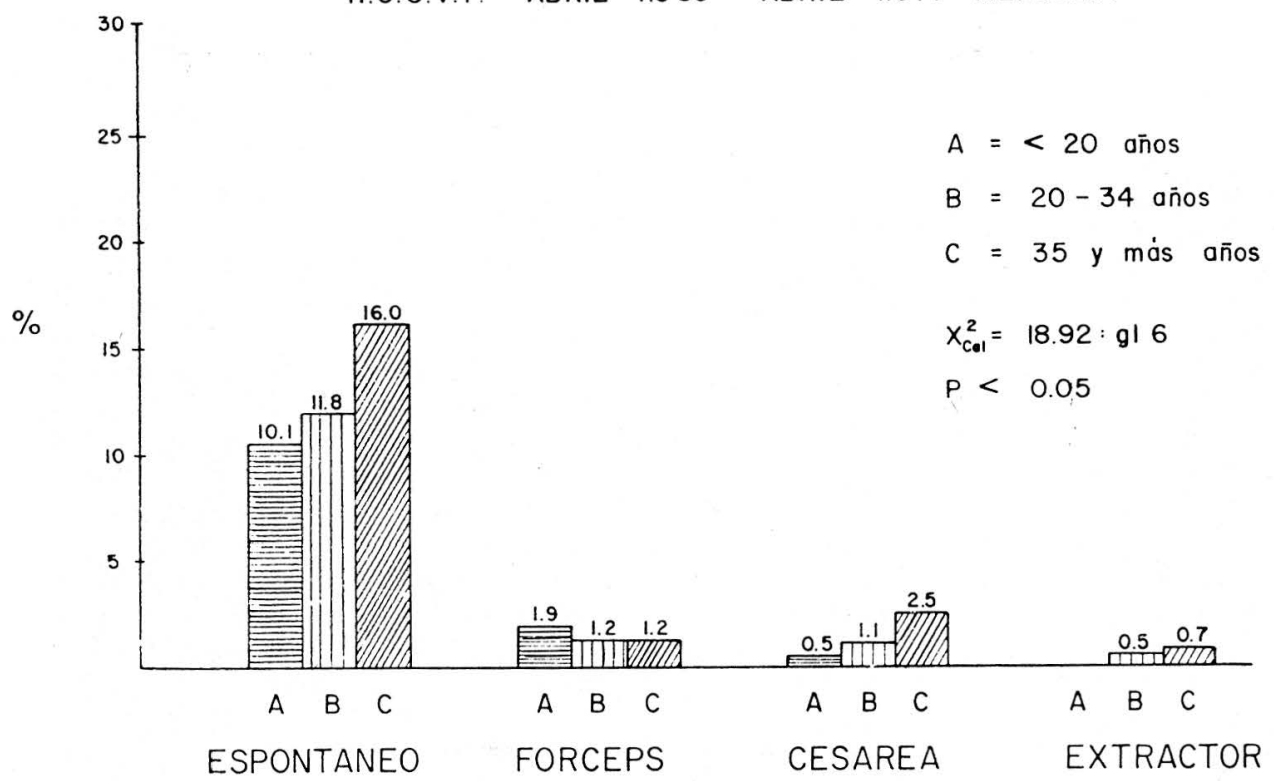




\section{RELACION ENTRE DURACION EN HORAS DEL PRIMER PERIODO $Y$ GRUPOS DE EDAD. - CASOS ANAL. 7.485} H.U.S.V.P. ABRIL 1.969 - ABRIL 1.971 MEDELLIN.

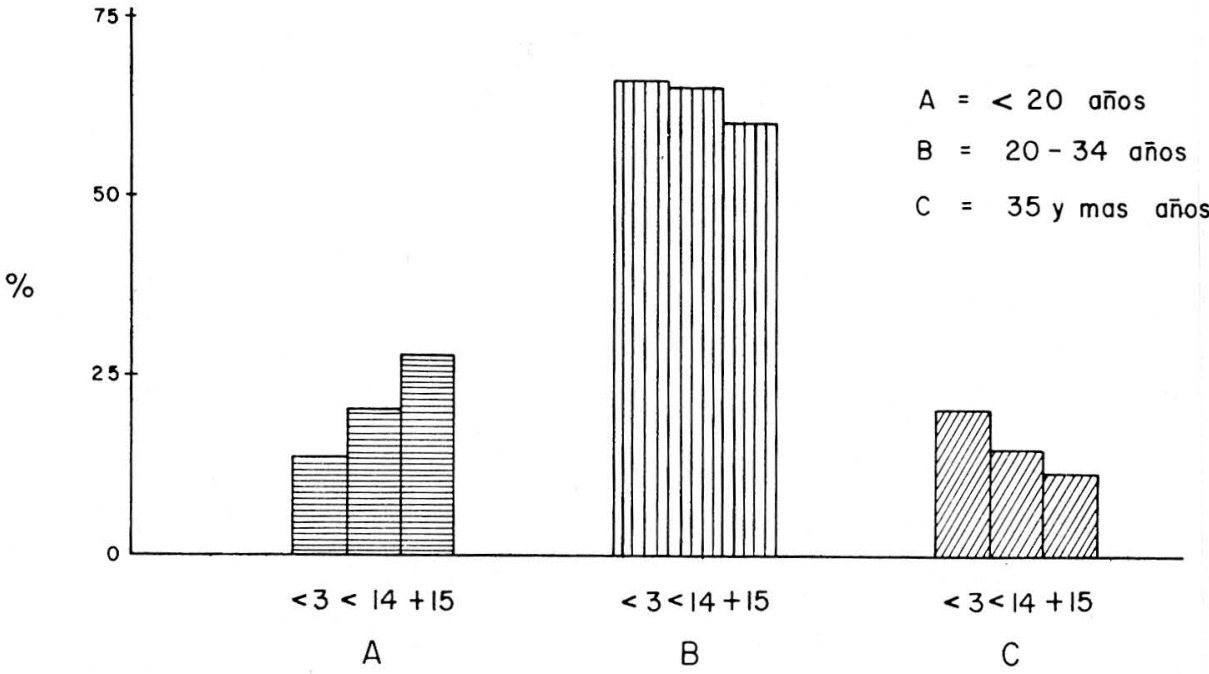

DURACION EN HORAS DEL PRIMER PERIODO

grupo de pacientes de 35 y más años, al $10 \%$ en el grupo de menores de 20. Esta proporción se invierte ligeramente en el grupo de partos con fórceps, pues en las mujeres jóvenes fue de $1,9 \%$ y en el de mayores de 35 años fue de 1,2\%. En el parto por cesárea la proporción fue nuevamente más alta en el grupo de mujeres de 35 y más años, $(2,5 \%)$, que en el de menores de $20(0,5 \%)$. En todos los tipos de parto, el grupo de madres de 20 a 34 años representa un valor intermedio en relación con los otros dos. La diferencia observada entre estos grupos de edad no es aleatoria.
7. Duración del primer período del parto. Observamos en el Gráfico № 3 cómo en el grupo A la duración del primer período del parto fue de más de 15 horas en una proporción alta de casos. En cambio en el grupo C fue de menos de 3 horas en una proporción mayor que en los otros dos. En el grupo B estuvo distribuída en forma igual en los tres grupos en los que se hizo la discriminación. Por la observación de este gráfico es posible pensar además que en el arupo de mujeres de menos de 20 años hubo una mayor proporción de pacientes que tuvieron trabajo prolongado, comparado con el de mayores 
Cuadro № 7

DURACION DEL SEGUNDO PERIODO DEL PARTO SE GUN EDAD DE LA MADRE. - CASOS ANAL. 7.5I5 H.U.S.V.P. ABRIL 1.969 - ABRIL 1.971 MEDELLIN

\begin{tabular}{c|c|c|c}
\hline $\begin{array}{c}\text { GRUPOS } \\
\text { DE EDAD }\end{array}$ & $<60^{\prime}$ & $60^{\prime}-120^{\prime}$ & $>120^{\prime}$ \\
\hline $\begin{array}{c}\text { A } \\
<0 \text { años }\end{array}$ & 97.3 & 2.3 & 0.4 \\
\hline $\begin{array}{c}\text { B } \\
20-34 \text { años }\end{array}$ & 98.1 & 1.7 & 0.2 \\
\hline $\begin{array}{c}\text { C } \\
35 \text { y mas años }\end{array}$ & 98.5 & 1.2 & 0.3 \\
\hline \begin{tabular}{c} 
TOTAL \\
\hline
\end{tabular}
\end{tabular}

de 35. Esto sin embargo es preciso investigarlo mejor.

8. Duración del segundo período del parto. Este período tuvo una duración menor de una hora en el $98 \%$ de los casos en todos los grupos de edad, como se observa en el Cuadro № 7. Esto se debió a la vigilancia médica a que estuvieron sometidas todas las pacientes. La duración mayor de una hora del segundo período, ocurrió en un porcentaje muy pequeño en todos los grupos.

9. Tipo de parto. De las 5.027 historias analizadas, vemos que en el $83 \%$ se informaron partos espontáneos, fórceps en el 10\%, cesáreas en el $5 \%$ y extractor al vacío sólo en el $2,5 \%$ (Cuadro No 8 ).

En el Gráfico № 4 se objetiva la diferencia entre los grupos en relación con la aplicación de fórceps y con la intervención cesárea. Tales diferencias son estadísticamente significativas, en lo que se refiere a la aplicación de fórceps en el grup oA, (18,9\%) comparado con el C $(3,2 \%)$ y a cesárea en este último $(11,2 \%)$ comparado con el A $(3,8 \%)$.

Si recordamos la frecuencia de aplicaciones de fórceps en el mismo período en el grupo total de pacientes, ( $10 \%$, Cuadro № 8 ), vemos cómo se duplicó en el de menores de 20 años. De la misma manera, la frecuencia 
Grafico № 4

PORCENTAJE DE PARTOS; SEGUN TIPO DE OCU RRENCIA Y EDAD DE LA MADRE. - CASOS ANAL. 5.027

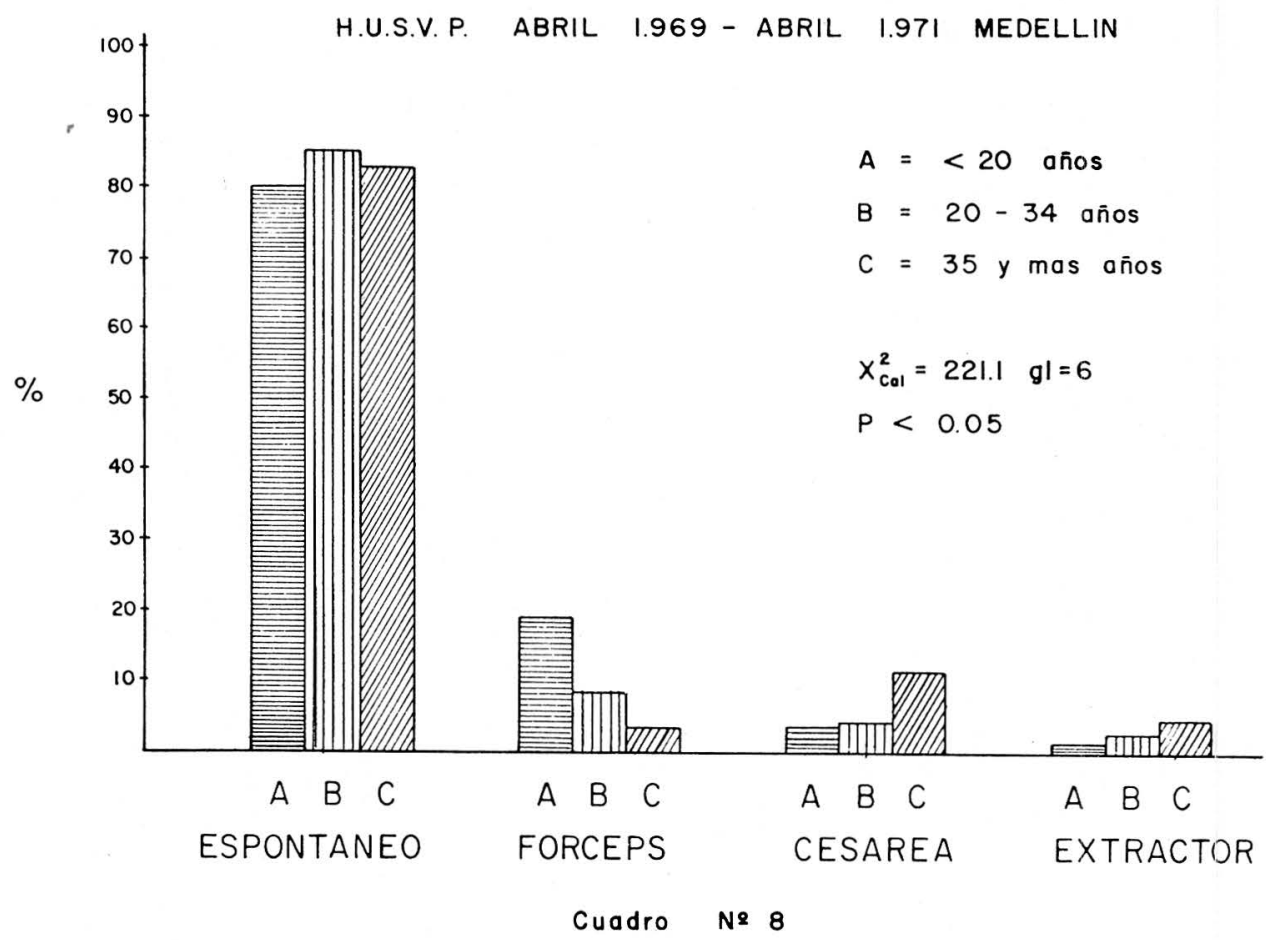

METODO DEL PARTO SEGUN EDAD DE LAS MADRES

EXPRESADO EN \%

H.U.S.V.P. ABRIL 1.969 - ABRIL I.97I MEDELLIN

\begin{tabular}{c|c|c|c|c|c}
\hline GRUPOS & ESPONTANEO & FORCEPS & CESAREA & EXTRACTOR & TOTAL \\
\hline A & 76 & 18.9 & 3.8 & 1.3 & 100 \\
B & 85.1 & 8.4 & 4 & 2.5 & 100 \\
C & 81.2 & 3.2 & 11.2 & 4.4 & 100 \\
\hline TOTAL & 82.6 & 9.8 & 5.1 & 2.5 & 100 \\
\hline
\end{tabular}


Grafico № 5

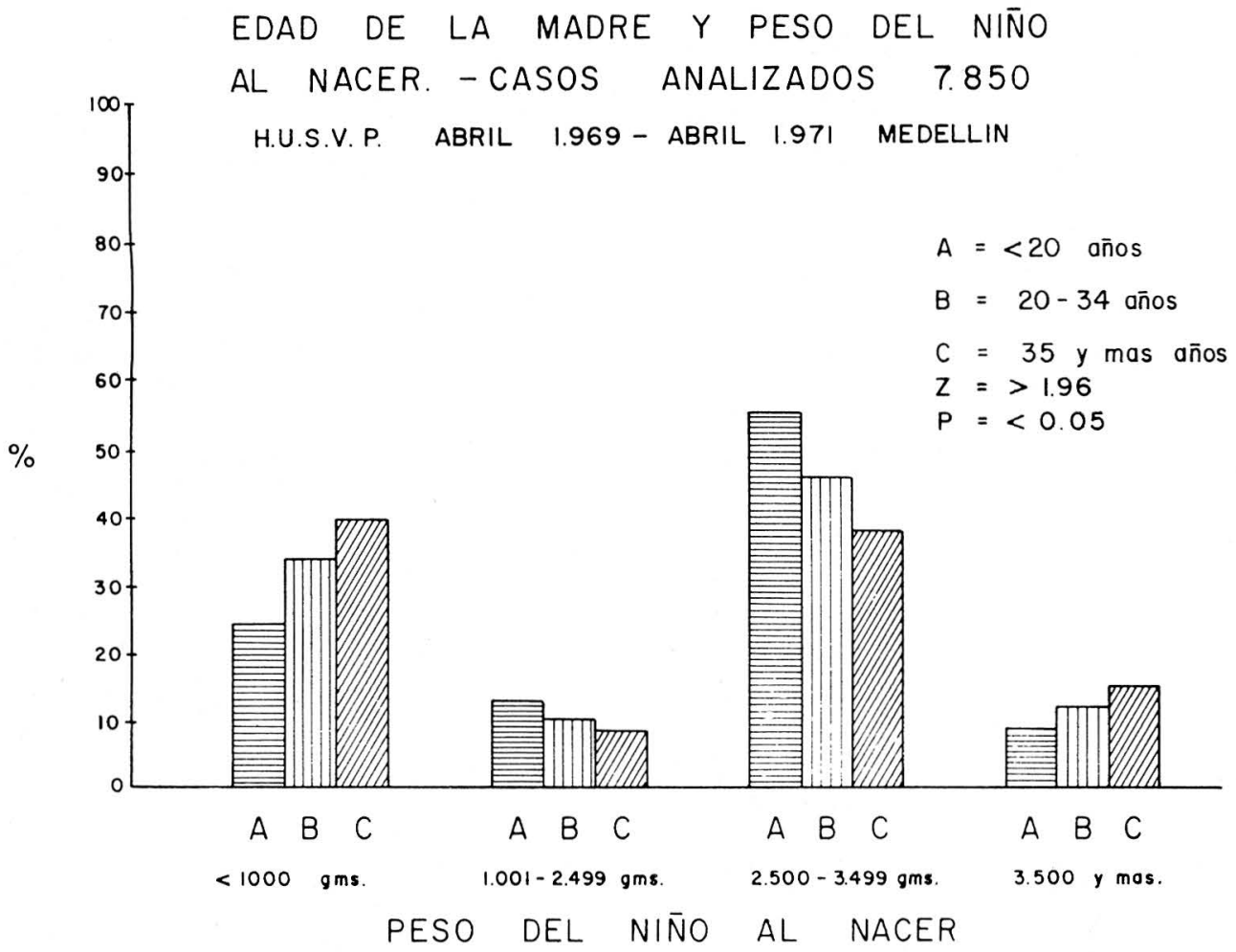

de intervenciones cesáreas en el grupo de pacientes mayores de 35 años fue el doble $(11,2 \%)$ de la encontrada para el grupo total ( $5 \%$ ).

10. Peso del niño al nacer. Al observar el Gráfico № 5 se encuentra que los niños con pesos menores de 1.000 gm., que en general son considerados como abortos, ocurrieron con una frecuencia mayor a medida que aumentó la edad de la madre, en razón de $1: 1,6$ entre el grupo $A$ y el C. (De $24 \%$ en madres jóvenes a $39,4 \%$ en las de más de 35 años).

En el siguiente grupo correspondiente a niños prematuros, que comprendieron aquellos cuyo peso estaba entre 1.000 y 2.499 gm., se observa que el mayor porcentaje lo presentaron las madres jóvenes (12,5\%); tal porcentaje fue descendiendo a medida que la edad de la madre aumentó ( $8,1 \%$ en las de más de 35 años).

En el grupo de niños de 2.500 a $3.499 \mathrm{gm}$., que se consideran los de peso normal, aunque el $55 \%$ de las madres jóvenes dieron a luz hijos dentro de este margen, este porcentaje descendió conforme aumentó la edad de la madre.

Las madres de más de 35 años tuvieron niños grandes, hecho que no fue $\tan$ frecuente en las de menos de 20. En nuestro estudio encontra- 
TASAS DE MUERTE FETAL SEgUN GRUPOS DE EDAD Y METODO DEL PARTO.-CASOS ANAL. 4.955 H.U.S.V.P. ABRIL 1.969 - ABRIL 1.971 MEDELLIN

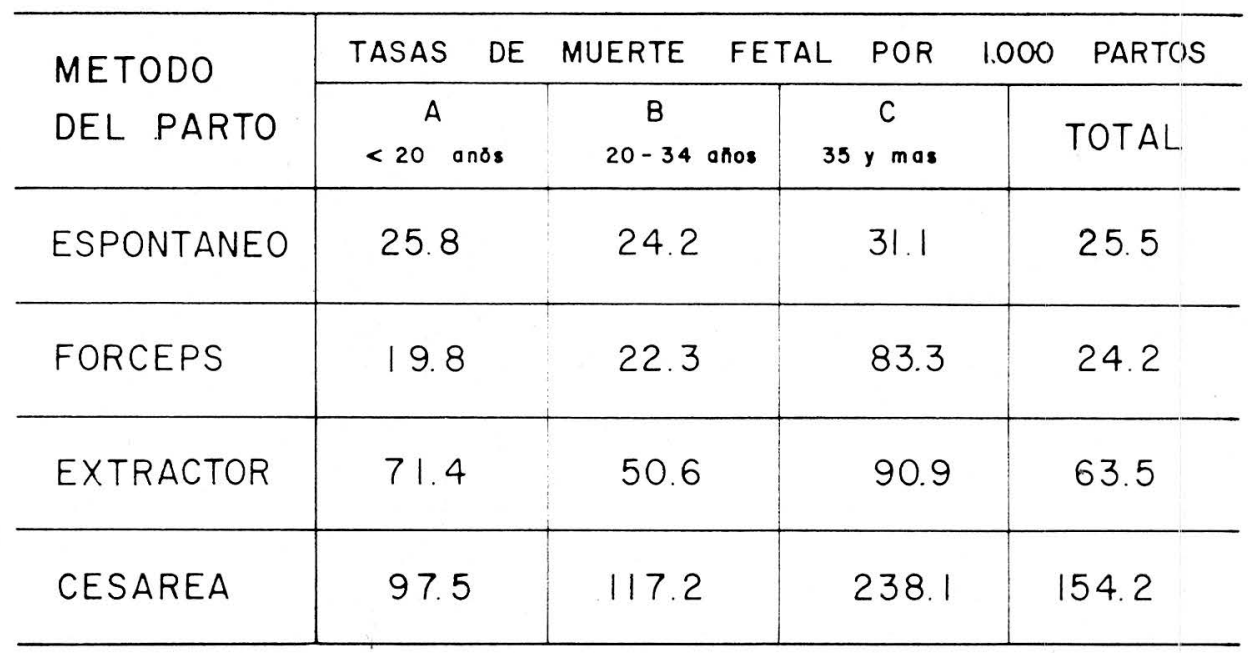

mos que el $15 \%$ de las madres de 35 y más años tuvo niños con más de $3.500 \mathrm{gm}$. de peso y sólo el $8 \%$ de las jóvenes los tuvo de ese peso. La diferencia proporcional entre estos grupos fue del orden del $73 \%$.

11. Mortalidad fetal. Al estudiar la muerte fetal en nuestro grupo de acuerdo con el método del parto (Cuadro No 9) y calculando la tasa por mil partos, encontramos que en la cesárea fue muy alta en el grupo A $(97,5 \times 1.000)$, pero aún lo fue mucho más en el $C$, en donde alcanzó la cifra de 238,1 x 1.000. El grupo B tuvo una cifra intermedia de $117,2 \times 1.000$. Son tan evidentes estas diferencias que de por sí indican una asociación marcada entre el ries- go de morir del feto extraído por cesárea, y el aumento de edad de la madre.

Otro hallazgo importante lo encontramos al comparar el grupo de madres mayores de 35 años con el resto de la población estudiada; las tasas de mortalidad son en ellas muy altas cualquiera que sea el método del parto y siempre las más bajas las encontramos en el grupo de madres de menor edad, lo que creemos que es consecuencia de las diferencias biológicas de las madres.

Al comparar las tasas de muerte fetal según el tipo de muerte y grupo de edad de las madres, (Cuadro № 10), observamos que la mortali- 
Cuadro Ne 10

TASAS DE MORTALIDAD FETAL SEGUN EDAD

DE LA MADRE. - CASOS ANALIZADOS 7.598

H.U.S.V.P. ABRIL 1.969 - ABRIL 1.971 MEDELLIN

\begin{tabular}{l|c|c|c|c}
\hline $\begin{array}{l}\text { MUERTE } \\
\text { FETAL }\end{array}$ & $\begin{array}{c}\text { A } \\
<20 \text { años }\end{array}$ & $\begin{array}{c}\text { B } \\
20-34 \text { años }\end{array}$ & $\begin{array}{c}\text { C } \\
35 \text { y más }\end{array}$ & TOTAL \\
\hline PRECOZ & 198.6 & 287.1 & 343.0 & 284.2 \\
\hline INTERMEDIA & 6.2 & 7.7 & 8.7 & 7.6 \\
\hline TARDIA & 14.7 & 15.5 & 31.6 & 18.0 \\
\hline
\end{tabular}

dad precoz fue muy alta en relación con la intermedia o tardía. Otro hecho que llama la atención es que, cualquiera que sea el tipo de muerte, tuvo una marcada tendencia a aumentar conforme aumentaba la edad de la madre, siendo muy marcado ese aumento en el grupo $C$ en relación con el $A$, pues se encontró una proporción en la muerte fetal precoz de $1: 1,7$, en la intermedia de $1: 1.4$, y en la tardía, de $1: 2,1$.

12. Infección puerperal. Se hizo una comparación de las tasas porcentuales de infección puerperal en los tres grupos de madres y se encontró que el riesgo es sensiblemente igual en todos, aunque de hecho llama la atención que esa tasa sea relativamente alta; en el grupo A es de 13.3, en el B de 12.6 y en el C de 12.5, lo que puede ser debido a la inclusión de los abortos en los tres grupos etarios.

\section{Conclusiones}

En el presente trabajo se ha puesto de presente cómo el comportamiento obstétrico de mujeres menores de 20 años es diferente en muchos aspectos al de las de 20 a 34 añas y al de las mayores de 35 .

Los hallazgos más importantes en el grupo de madres menores de 20 
años son los siguientes: es más alta la incidencia de toxemia, el primer período del parto parece mostrar una tendencia a tener una duración mayor, hay una mayor frecuencia en el uso del fórceps y la ocurrencia de niños prematuros es mayor.

Ese mismo grupo de mujeres es el que presenta una menor frecuencia de membranas rotas en el parto espontáneo. También en ese grupo la mortalidad fetal es la más baja.

En el grupo de mujeres de 20 a 34 años es donde la toxemia muestra la tasa más baja, y este es el hecho saliente de este grupo.

En cambio, el grupo de madres de 35 y más años presenta ruptura de membranas en una proporción más alta que los otros dos; el primer período del parto tiene tendencia a ser de corta duración; la intervención cesárea es más frecuente que en los otros dos grupos y hay niños grandes en una mayor proporción de casos.

Los tres grupos presentan un hecho común: la ocurrencia de ruptura de membranas es alta en todos ellos. Los abortos del 1: y 2 : trimestres y la mortalidad fetal aumentan conforme aumenta la edad de la madre.

\section{Resumen}

Se presenta un estudio sobre el comportamiento obstétrico de mujeres menores de 20 años y se compara con el de mujeres de 20 a 34 años y con el de 35 y más, basado en el análisis de 7.897 historias clínicas de pacientes atendidas en el Hospital Universitario San Vicente de Paúl de Medellín, Colombia, en un período de 25 meses, entre Abril de 1969 y Abril de 1971.

\section{Summary}

In this study, the obstetric performance of three groups of patients: less than 20 years of age, 20-34 and 35 and over, has been analysed. For this purpose, 7.897 histories, covering a period of 25 months of the obstetric service of San Vicente de Paul University Hospital in Medellin, Colombia, have been perused. The main findings are these:

1. In the younger patients (20 years of age and less) there is a higher toxemia rate, the first period of labor has a tendency to be of more duration, there is a higher incidence of forceps application and the prematurity rate is higher. In this group of patients the incidence of ruptured membranes is lower in spontaneous labor. Also the fetal mortality is low.

2. The main fact in the group of patients between 20 and 34 years of age is the low incidence of toxemia.

3 . In the group of patients of more than 35 years of age the spontaneous rupture of membranes is higher, the first period of labor shows a tendency to be of short duration, and the cesarean section rate is more elevated than in the other two groups.

4. The three groups have a fact in common: the high incidence of ruptured membranes in all of them.

5. The first and second trimester abortion rate increases with the age of the mother. The same thing is observed with the fetal mortality.

\section{BIBLIOGRAFIA}

1 Estudio de Recursos Humanos para la Salud y Educación Médica en Colombia. Investigación Nacional de Morbilidad. Ministerio de Salud Pública. Asociación de Facultades de Medicina. P.E.S. Bogotá, 1968. 
2 CLAMAN, A. D. \& BELL, H. M.: Pregnancy in very young teen-ager. Am. J. Obst. \& Gynec. 90: 350 (Oct. 1), 1964.

3 RUSSEL, J. K. Pregnancy in the young teen-ager. The Lancet. 1: 365 (Feb. 15), 1969.

4 BATTAGLIA, F. C., FRAZIER, T. M. \& HELLEGERS, A. E.: Obstetric and Pediatric Complications of Juvenile Pregnancy. Pediatrics. 32: 902 (Nov.) 1963.

5 HULKA, J. F. \& SCHAAF, J. T. Obstetrics in adolescents: a controlled study of deliveries by mothers 15 years of age and under. Obst. \& Gynec. 23: 678 (May) 1964.

6 SARREL, P. M. \& DAVIS, C. D. The young unwed primipara. Am. J. Obst. \& Gynec. 95: 722, (Jul. 1), 1966.

7 ISRAEL, S. L. \& WOUTERSZ, T. B.: Teen age obstetrics. Am. J. Obst. \& Gynec. 85: 659. (Mar. 1), 1963.

8 HASKINS, A. L.: Discusión del artículo anterior. Am. J. Obst. \& Gynec. 85: 666. (Mar 1), 1963.

9 POLIAKOFF, S. R.: Pregnancy in the young primigravida. Am. J. Obst. \& Gynec. 76: 746 (Oct.), 1958.

10 MUSSIO, T. J.: Primigravidas under age 14. Am. J. Obst. \& Gynec. 84: 442 (Aug. 15), 1962.

11 GABRIELSON, I. W., KLERMAN, L. V., CURRIE， J. B., TYLER, N. C., JEKEL, J. F.
Suicide attempts in a population pregnant as teen agers. Amer. J. Publ. Health 60: 2289 (Dec.), 1970.

12 SEMMENS, J. P. \& MCCLAMORY, J. C.: Teen age pregnancies. Obst. \& Gynec. 16: 31, (Jul.), 1960.

13 KEEVE, J. P.: Selected social, educational and medical characteristics of primiparous 12-16 years old girls. Pediatrics. 36: 394, (Sept.), 1965.

14 ISRAEL, S. L. \& DEUTSCHBERGER, J.: Relation of the mother's age to obstetric performance. Obst. \& Gynec. 24: 411 (Sept.) 1964.

15 CASTELLANOS, R., AGUERO, O., MORENO, L. F.: Estudio sobre mil madres adolescentes menores de 15 años. Rev. Obst. Gin. Venezuela, 19: 65, 1969.

16 SARREL, P. M. \& KLERMAN, L. V.: The young unwed mother. Obstetric results of a program of comprehensive care. Am. J. Obst. \& Gynec. 105: 575 (Oct. 15), 1969.

17 Clasificación Internacional de Enfermedades. Organización Panamericana de la Salud. Publicaciones Científicas. No 52. Washington. D. C. 1961.

18 GAVIRIA, B., BOTERO, J., OCHOA, G., RESTREPO, L. F., BUSTOS, R., CALLE, S., MONTOYA, M., VASQUEZ, D., PINEDA, D. : Letalidad Perinatal. Rev. Colomb. Obstet. Ginecol. 11: 339, 1960. 